\title{
Decreased Threshold of Fasting Serum Glucose for Cardiovascular Events: MASHAD Cohort Study
}

Fatemeh Sadabadi\# ${ }^{1}$, Aida Gholoobi\# ${ }^{2}$, Alireza Heidari-Bakavol\# ${ }^{3}$, Mohsen Mouhebati ${ }^{\sharp 3}$, Ali Javandoost ${ }^{1}$, Zahra Asadi ${ }^{1}$, Maryam Saberi-Karimian ${ }^{1}$, Susan Darroudi ${ }^{1}$, Mohammad Sadegh Khorrami Mohebbseraj ${ }^{4}$, Farzad Rahmani ${ }^{1}$, Najmeh Malekzadeh Gonabadi ${ }^{5}$, Fatemehalsadat Jafari Sheshtamad ${ }^{1}$, Sara Samadi ${ }^{1}$, Afsane Bahrami ${ }^{6}$, Gordon Ferns ${ }^{7}$, Majid Ghayour-Mobarhan ${ }^{* 1,8}$, Habibollah Esmaeili*9

\begin{abstract}
Background: Cardiovascular disease (CVD) is the leading cause of morbidity and mortality globally, and specifically in Iran. Generally, diabetes mellitus is the result of impaired glucose tolerance which together with dyslipidemia are considered as important risk factors of CVD.

The aim of this study was to determine the relationship between fasting serum glucose (FSG), lipid profile and CVD endpoints, and to establish an optimal FSG cut-off in the MASHAD cohort study after nearly 6 years of follow-up.

Methods: All the participants of MASHAD study were followed up for 6 years to determine their cardiovascular status. FSG, fasting lipids, and physical examinations were all recorded. To identify the optimal cut- off point of FSG, we carried out receiver operating curve (ROC) analysis

Results: We determined MASHAD cutoff point of blood glucose as $90 \mathrm{mg} / \mathrm{dl}$ predicting the CVD outcome. The sensitivity and specificity of the FSG criterion were $54.34 \%$ and $71.68 \%$, respectively. The AUC was $0.665(95 \%$ CI 0.656-0.675, P<0.0001). The adjusted hazard ratio show that FSG is associated with 2.34 increase in CVD risk using MASHAD cutoff point (HR 2.34, 95\% 1.73-3.17, $\mathrm{P}<0.001)$.

Conclusions: These findings suggest that not only FSG and lipid profile are related to CVD outcome in the MASHAD study, but also elevated fasting glucose levels is strongly associated with cardiovascular events in this population. Besides, the fasting glucose at a threshold of $90 \mathrm{mg} / \mathrm{dl}$ can be used for screening cardiovascular events among the Iranian population.
\end{abstract}

Keywords: Fasting Serum Glucose, Lipid profile, Cardiovascular disease (CVD), MASHAD cohort study.

1: Metabolic Syndrome Research Center, School of Medicine, Mashhad University of Medical Sciences, Mashhad, Iran.

2: Medical Genetics Research Centre, School of Medicine, Mashhad University of Medical Sciences, Mashhad, Iran;

3: Cardiovascular Research Center, School of Medicine, Mashhad University of Medical Sciences, Mashhad, Iran.

4: Department of Medical Genetic and Molecular Medicine, School of Medicine, Mashhad University of Medical Sciences, Mashhad, Iran.

5: Department of genetic, School of science, Sistan and Baluchestan University, Zahedan, Iran.

6: Cellular and Molecular Research Center, Birjand Univensity of Medical Sciences, Birjand, Iran.

7: Department of Medical Education, Brighton \& Sussex Medical School, Brighton, BN1 9PH, UK

8: International UNESCO Center for Health-Related Basic Sciences and Human Nutrition, Department of Nutrition, School of Medicine, Mashhad University of Medical Sciences, Mashhad, Iran.

9: Department of Biostatistics \& Epidemiology, School of Health, Management \& Social Determinants of Health Research Center, Mashhad

University of Medical Sciences, Mashhad, Iran.

\#These authors equally contributed to this study as first authors.

*Corresponding author: Majid Ghayour-Mobarhan; Tel: +985138002288; E-mail: ghayourm@mumsacir

\& Habibollah Esmaeily; Tel: +98155164156; E-mail: Esmailyh@mumsac.ir.

Received: 14 Jan, 2020; Accepted: 2 Feb, 2020 


\section{Introduction}

Cardiovascular disease (CVD) is the leading cause of morbidity and mortality globally, and in Iran there has been a rising prevalence of CVD (1). Impaired glucose tolerance often progresses to overt diabetes mellitus, and is often associated with dyslipidemia, both important risk factors for CVD (2). An impaired fasting glucose (IFG) is also reported to be associated with an increased risk of CVD (3). There has been considerable controversy about the diagnostic threshold of the FPG and the reduction of the cut-off for IFG from $110 \mathrm{mg} / \mathrm{dl}$ to $100 \mathrm{mg} / \mathrm{dl} \mathrm{(4).} \mathrm{However,} \mathrm{this}$ threshold may vary for different populations. There has been a single study that has investigated the FPG cut-off threshold for determining a high risk of cardiovascular risk in the Iranian population (5), but there have been no studies that have assessed the FSG threshold for CVD outcomes in this population.

We aimed to determine the relationship between fasting serum glucose (FSG) and lipid profile and CVD endpoints, and to establish an optimal FSG cut-off in the MASHAD cohort study after nearly 6 years of follow-up.

\section{Materials and methods}

The study design of the MASHAD Study has been described previously (6). In brief, this cohort study began in 2007 with 9704 participants aged 35-65 years and will continue until 2020. The follow-up being accomplished every 3 years. Informed consent was obtained from all individuals recruited to the study. The demographic data, anthropometric measurements, lifestyle data included smoking habits (non, ex- or current smoker) and education level, also cardiovascular risk-related questionnaire was collected. At baseline, all subjects were free of CVD.

All the participants were followed up to assess their cardiovascular outcomes. This follow-up occurred between 2015-2016, during which patients who reported symptoms of CVD were asked to attend for a cardiac examination, and to bring with them any related medical documents.

They attended after fasting at least 12 hours in order to take blood samples and biochemical parameters were assessed.
Recumbent blood pressure and electrocardiography (ECG) were performed on all participants, and each was examined by a cardiologist. CVD events were confirmed, based on the history, previous clinical tests, and medical examinations including stress echocardiography, radioisotope, angiography, CT angiography and exercise tolerance test (ETT) and relevant available medical records, and were adjudicated by an electrophysiologist and two interventional cardiologists.

We excluded stroke patients from our study since some patients with a stroke had died and some were reluctant to be examined, nor did we have access to a MRI to confirm the diagnosis in all these patients.

To identify the optimal cutoff point of FSG as a predictor of the CVD events in the Mashhad study, we undertook receiver operating curve (ROC) analysis.

\section{Statistical analysis}

SPSS version 18 (SPSS Inc, IL, USA) was used for all statistical analyses. The hazard ratios (HRs) for CVD outcomes between individuals above and below the MASHAD and Europeans cutoff points were estimated using cox proportional hazard models. MedCalc statistical software 17.2 was used to perform ROC curve analysis. The level of statistical significance was set at $p<0.05$.

\section{Results}

\section{Study population}

From 9704 participants (mean age 48.07 \pm 8.26 years), 235 subjects were confirmed as having a positive CVD outcome from 2015 to 2016. Of these, 27 patients had died of a cardiac cause. FSG, TG, cholesterol, LDL-C, anthropometric indices and demographic data were significantly higher in subjects with CVD compared to those without (Table 1).

\section{ROC graph analysis}

Generally, ROC analysis and the area under the curve (AUC) describes the discrimination between diseased and normal cases at the best threshold for a diagnostic marker (7). As 
shown in Fig. 1, we determined that the optimum cut-off point of serum glucose as $90 \mathrm{mg} / \mathrm{dl}$ in predicting CVD outcomes in the MASHAD population sample. The sensitivity and specificity of the FSG criterion $(90 \mathrm{mg} / \mathrm{dl})$ were $54.34 \%$ and $71.68 \%$, respectively. The AUC was 0.665 ( $\mathrm{p}<$ $0.0001)$.

\section{FSG and lipid profile of the MASHAD cohort} study before and after CVD outcome

Table 2 summarized the comparison of the changes in biochemical features at baseline and follow up of participants with and without CVD. The results show that the serum FSG $(\mathrm{p}=0.006)$, cholesterol ( $\mathrm{p}<0.001)$, and LDL-C increased significantly in participants with CVD ( $p<$ $0.001)$. There were no significant changes in serum TG and HDL-C between baseline and follow up in patients with and without CVD.

\section{Hazards Ratios for CVD according to FSG}

In Table 3, Cox's proportional hazard models were used to estimate CVD outcomes according to the cut points of blood glucose of European (proposed by IDF and World Health Organization (WHO)) (8) and the MASHAD study. The HR for CVD events showed that a FSG above the threshold was associated with more than a 3-fold increase in risk. Moreover, the unadjusted HR was approximately similar in two groups. There were no significant changes after adjustment for variables such as sex, age, dyslipidemia, hypertension, BMI and smoking status mentioned in the models 2-6.

Table 1. Fasting Glucose, lipid profile and anthropometric data of patients with CVD compared to the rest of the population.

\begin{tabular}{|c|c|c|c|c|}
\hline & & CVD event $N=235$ & Without CVD event $N=8078$ & p value \\
\hline Age (year) & & $54.22 \pm 6.99$ & $47.92 \pm 8.23$ & 0.001 \\
\hline & Male & $105(46.7)$ & $3840(40)$ & 0.026 \\
\hline & Female & $120(53.3)$ & $5767(60)$ & \\
\hline BMI (kg/m2) & & $28.79 \pm 4.50$ & $27.88 \pm 4.74$ & 0.004 \\
\hline Waist circumfe & nce (cm) & $98.87 \pm 10.10$ & $95.14 \pm 12.07$ & 0.001 \\
\hline Hip circumfere & ee $(\mathrm{cm})$ & $104.10 \pm 9.38$ & $103.72 \pm 9.33$ & 0.542 \\
\hline TG (mg/dl) & & $139.00(100.25-217.50)$ & $120.00(84.00-171.00)$ & 0.001 \\
\hline Cholesterol (mg & & $199.38 \pm 42.63$ & $191.15 \pm 39.04$ & 0.002 \\
\hline HDL-C (mg/dl) & & $40.08 \pm 9.10$ & $42.93 \pm 9.96$ & 0.001 \\
\hline LDL-C (mg/dl) & & $121.97 \pm 35.63$ & $116.40 \pm 35.29$ & 0.020 \\
\hline FSG (mg/dl) & & $118.35 \pm 62.15$ & $92.02 \pm 38.31$ & 0.001 \\
\hline Education level & $\begin{array}{l}\text { Under high } \\
\text { school diploma }\end{array}$ & $7505(97.2)$ & $214(2.8)$ & 0.210 \\
\hline $\mathrm{n}(\%)$ & $\begin{array}{l}\text { Above high } \\
\text { school diploma }\end{array}$ & $989(97.9)$ & $21(2.1)$ & \\
\hline & non & $5916(97.7)$ & $142(2.3)$ & \\
\hline Smoking n (\%) & ex & $827(95.3)$ & $41(4.7)$ & \\
\hline & current & $1789(97.2)$ & $52(2.8)$ & \\
\hline
\end{tabular}

BMI body mass index, LDL-C low-density lipoprotein, HDL-C high-density lipoprotein cholesterol.

Values are expressed as mean $\pm \mathrm{SD}$ and median. 
Table 2. Comparison of the changes in biochemical features at baseline and follow up in participants with and without CVD.

\begin{tabular}{|c|c|c|c|c|c|c|c|}
\hline & \multicolumn{2}{|c|}{$\begin{array}{l}\text { Patients with } \\
\text { CVD }(\mathrm{N}=235)\end{array}$} & \multicolumn{4}{|c|}{$\begin{array}{l}\text { Subjects without } \\
\text { CVD }(\mathrm{N}=411)\end{array}$} & \multirow[b]{2}{*}{ p value } \\
\hline & Baseline & $\begin{array}{l}\text { Follow } \\
\text { up }\end{array}$ & $\begin{array}{l}\text { Difference at } \\
\text { follow-up }\end{array}$ & Baseline & $\begin{array}{l}\text { Follow } \\
\text { up }\end{array}$ & $\begin{array}{l}\text { Difference at } \\
\text { follow-up }\end{array}$ & \\
\hline $\begin{array}{l}\text { FSG } \\
(\mathrm{mg} / \mathrm{dl})\end{array}$ & $\begin{array}{l}118.96 \pm \\
60.14\end{array}$ & $\begin{array}{l}151.06 \\
\pm 77.20\end{array}$ & $32.09 \pm 64.02$ & $\begin{array}{l}93.92 \pm \\
39.74\end{array}$ & $\begin{array}{l}121.52 \pm \\
63.29\end{array}$ & $27.59 \pm 56.55$ & 0.006 \\
\hline p value & $<0.001$ & & & $<0.001$ & & & \\
\hline TG (mg/dl) & $\begin{array}{l}139 \\
(101.25- \\
215.50) \\
\end{array}$ & $\begin{array}{l}143 \\
(111.5- \\
190.5) \\
\end{array}$ & $-4.26 \pm 87.48$ & $\begin{array}{l}124 \\
(86-178)\end{array}$ & $\begin{array}{l}134 \\
(100.75- \\
196) \\
\end{array}$ & $3.42 \pm 105.23$ & 0.211 \\
\hline p value & 0.532 & & & 0.565 & & & \\
\hline $\begin{array}{l}\text { Cholesterol } \\
\text { (mg/dl) }\end{array}$ & $\begin{array}{l}197.45 \pm \\
43.48\end{array}$ & $\begin{array}{l}203.55 \\
\pm 50.34\end{array}$ & $6.09 \pm 55.68$ & $\begin{array}{l}192.27 \pm \\
42.48\end{array}$ & $\begin{array}{l}219.88 \pm \\
53.50\end{array}$ & $27.60 \pm 55.52$ & $<0.001$ \\
\hline p value & 0.161 & & & $<0.001$ & & & \\
\hline $\begin{array}{l}\text { HDL-C } \\
\text { (mg/dl) }\end{array}$ & $\begin{array}{l}40.26 \pm 8 \\
99\end{array}$ & $\begin{array}{l}43.81 \pm \\
11.50\end{array}$ & $3.54 \pm 12.41$ & $\begin{array}{l}42.52 \pm \\
9.44\end{array}$ & $\begin{array}{l}48.28 \pm \\
11.30\end{array}$ & $5.76 \pm 10.98$ & 0.210 \\
\hline p value & $<0.001$ & & & $<0.001$ & & & \\
\hline $\begin{array}{l}\text { LDL-C } \\
(\mathrm{mg} / \mathrm{dl}) \\
\end{array}$ & $\begin{array}{l}121.78 \pm \\
36.56\end{array}$ & $\begin{array}{l}130.05 \\
\pm 45.86\end{array}$ & $8.26 \pm 47.98$ & $\begin{array}{l}113.71 \pm \\
38.88\end{array}$ & $\begin{array}{l}141.50 \pm \\
42.85\end{array}$ & $27.78 \pm 45.48$ & $<0.001$ \\
\hline p value & 0.030 & & & $<0.001$ & & & \\
\hline
\end{tabular}

* Changes in biochemical features at baseline and follow up in patients with and without CVD adjusted for age and sex using general linear model and repeated measurements.

Table 3. Hazard Ratios of CVD outcomes according to the FSG of MASHAD cohort study participants at baseline.

Europeans cut point $(>100)(\mathbf{m g} / \mathbf{d l})$

\begin{tabular}{cccccc}
\hline $\begin{array}{c}\text { Cox } \\
\text { proportional } \\
\text { hazard models }\end{array}$ & HR $(95 \%$ CI) & p value & $\begin{array}{c}\text { Cox } \\
\text { proportional } \\
\text { hazard models }\end{array}$ & HR (95\% CI) & p value \\
\hline Model 1 & $3.89(2.91-5.21)$ & $<0.001$ & Model 1 & $3.40(2.52-4.54)$ & $<0.001$ \\
\hline Model 2 & $2.85(2.12-3.83)$ & $<0.001$ & Model 2 & $2.58(1.92-3.48)$ & $<0.001$ \\
\hline Model 3 & $2.78(2.06-3.75)$ & $<0.001$ & Model 3 & $2.54(1.88-3.42)$ & $<0.001$ \\
\hline Model 4 & $2.79(2.07-3.76)$ & $<0.001$ & Model 4 & $2.55(1.89-3.44)$ & $<0.001$ \\
\hline Model 5 & $2.57(1.91-3.47)$ & $<0.001$ & Model 5 & $2.36(1.75-3.19)$ & $<0.001$ \\
\hline Model 6 & $2.55(1.88-3.44)$ & $<0.001$ & Model 6 & $2.34(1.73-3.17)$ & $<0.001$
\end{tabular}

CI, confidence interval; HR, hazard ratio.

Model 1, Unadjusted; Model 2, Adjusted for sex and age; Model 3, Adjusted for sex, age and dyslipidemia; Model 4, Adjusted for sex, age, dyslipidemia and smoking status; Model 5, Adjusted for sex, age, dyslipidemia, smoking status and hypertension; Model 6, Adjusted for sex, age, dyslipidemia, smoking status, hypertension and BMI. 


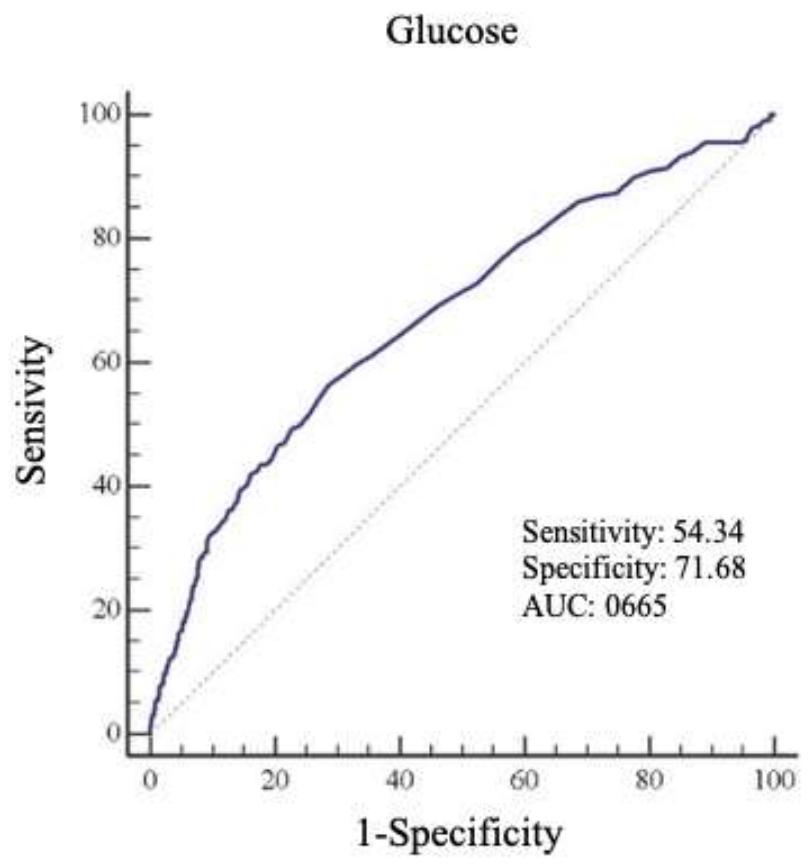

Fig. 1. ROC curve of FSG for detecting CVD outcomes in the MASHAD study. The $90 \mathrm{mg} / \mathrm{dl}$ cut-off value for fasted serum glucose gave the optimal sensitivity plus specificity for predicting CVD outcomes.

\section{Discussion}

Few studies have investigated the cardiovascular outcomes according to different cut-offs for fasting serum glucose levels $(1,2)$.

Different ethnic groups vary in their susceptibility to CVD, and different glycemic thresholds have been used in different populations. These cut-offs differ for the populations studied. On the other hand, data for tracking these thresholds in various ethnicities and races is limited (5); hence, it is of importance that optimal fasting glucose cut-offs are identified for the Western Asian population.

The association between FSG with CVD risk factors has been reported previously (3), and is consistent with our findings. Our findings support the study of Shaye et al. who have also reported that systolic blood pressure, family history of CVD, TG, and LDL-C levels are associated with elevated fasting glucose levels and a high normal range of FSG may help to predict CVD event. They also showed CVD risk was higher in individuals who had high "normal range' of FPG (95-99 $\mathrm{mg} / \mathrm{dl})$ compared to the subjects with FPG levels $<80 \mathrm{mg} / \mathrm{dl}$, (HR 1.53; CI 95\% [1.22-1.91], p< 0.001) (9).
Our findings also support the results of a study among Korean adults in which it was shown that IFG can predict CVD events. In this study, age-adjusted HR for CVD were 1.17 (95\% CI 1.13-1.20) for subjects with FSG 100-109 $\mathrm{mg} / \mathrm{dl}, 1.30$ (1.24-1.35) for subjects with FSG $110-125 \mathrm{mg} / \mathrm{dl}$, and $1.81(1.75-1.86)$ for diabetic participants (4).

TG changes were statistically significant between baseline and follow-up, after a CVD, however in the current study we did not find changes in TG one reason for this may be that $60.2 \%$ of patients who had cardiovascular event, were placed on statin therapy which in addition to reducing serum LDL cholesterol, also has a modest effect on serum TG (10). Furthermore, serum fasted TG values are also affected acutely following a $\mathrm{CV}$ event, and then may return to normal. We did not specify the time interval after an event in the follow-up protocol.

There were no significant differences in changes of HDL-C at baseline and follow up in patients with and without CVD adjusted for age and sex. Briel et al. in their meta-regression analysis suggested that the risk of coronary 
heart disease does not change significantly with increasing circulating HDL-C (11), which is in agreement with our study.

The most important finding of the current study was that an optimal cut-off value for fasting serum glucose concentrations for predicting CVD events in our population was lower $(90 \mathrm{mg} / \mathrm{dl})$ compared to the ADA Expert Committee recommendations (12). It was found that CVD risk was substantially higher in our population who had FSG of $90 \mathrm{mg} / \mathrm{dl}$, and this increased risk was similar to those at the European threshold $(100 \mathrm{mg} / \mathrm{dl})$. Esteghamati et al. found that cardiovascular risk factors increased at a FPG of 90-95 mg/dl, as our data indicated (5).

Huang et al. assessed the optimal FPG cut off points for predicting metabolic syndrome (MetS) and further type 2 diabetes (T2D) and CVD in an aged Taiwanese population with normal FPG values. FPG Cut-off points obtained for MetS were $93 \mathrm{mg} / \mathrm{dl}$ for males and $92 \mathrm{mg} / \mathrm{dl}$ for females. The group with high FPG had a higher incidence rate of CVD for females (HR: 1.24, 95\% CI: $1.015-1.515, \mathrm{p}=0.035$ ) (13). The current study also showed an incidence rate of CVD that increased 2.55 and 2.34-fold in the group with a high-FPG compare to the group with a FSG of $<90 \mathrm{mg} / \mathrm{dl}$, according to the FSG

\section{References}

1. Nejat A, Mirbolouk M, Mohebi R, Hasheminia M, Tohidi M, Saadat N, et al. Changes in lipid measures and incident coronary heart disease: Tehran Lipid \& Glucose Study. Clin biochem. 2014;47(13-14):1239-44.

2. Emerging Risk Factors Collaboration; N Sarwar, P Gao, S R Kondapally Seshasai, R Gobin, et al. Diabetes mellitus, fasting blood glucose concentration, and risk of vascular disease: a collaborative meta-analysis of 102 prospective studies. Lancet. 2010;375(9733):2215-22.

3. Mongraw-Chaffin M, LaCroix AZ, Sears DD, Garcia L, Phillips LS, Salmoirago-Blotcher E, et al. A prospective study of low fasting glucose with cardiovascular disease events and all-cause cut points of European and MASHAD cohort study, respectively in both genders.

Chundan Bao et al. described the optimum cut-off of FPG for past undiagnosed diabetes are $5.6 \mathrm{mmol} / \mathrm{l}(100.8 \mathrm{mg} / \mathrm{dl})$ (AUC of FPG 0.93; sensitivity $82.0 \%$ in screening unusual glucose metabolism)(14).

These findings suggest that an elevated fasting glucose and lipid profile is strongly associated with cardiovascular events in population of MASHAD study.

A threshold fasting glucose of $90 \mathrm{mg} / \mathrm{dl}$ may be used for screening cardiovascular risk among the Iranian population, lower than for other population groups.

The fasting glucose at a threshold of $90 \mathrm{mg} / \mathrm{dl}$ may be used for screening for an increased risk of cardiovascular events among the Iranian population.

These findings need to be tested over a longer time period for CVD follow up and other populations that have different environmental and genetic backgrounds, so that stratified data can be obtained by gender and socioeconomic group.

\section{Acknowledgment}

We thank all the patients and their family members who volunteered to participate in this study.

mortality: The Women's Health Initiative. Metabolism. 2017;70:116-124.

4. Kim H-K, Kim C-H, Kim EH, Bae SJ, Choe J, Park J-Y, et al. Impaired fasting glucose and risk of cardiovascular disease in Korean men and women: the Korean Heart Study. Diabetes Care. 2013;36(2):328-35.

5. Esteghamati A, Zandieh A, Hafezi-Nejad N, Sheikhbahaei S, Abbasi M, Gouya MM, et al. Revising the fasting glucose threshold for detection of cardiovascular risk factors: analysing two representative population-based studies of more than 50000 Iranians in 3 years: The National Survey of Risk Factors for Non-Communicable Diseases of Iran. Ann hum biol. 2015;42(2):150-8. 
6. Ghayour-Mobarhan M, Moohebati M, Esmaily H, Ebrahimi M, Parizadeh SMR, Heidari-Bakavoli AR, et al. Mashhad stroke and heart atherosclerotic disorder (MASHAD) study: design, baseline characteristics and 10year cardiovascular risk estimation. Int J Public Health. 2015;60(5):561-72.

7. Perkins NJ, Schisterman EF. The Inconsistency of "Optimal"Cutpoints Obtained using Two Criteria based on the Receiver Operating Characteristic Curve. Am J Epidemiol. 2006;163(7):670-5.

8. Aschner P. New IDF clinical practice recommendations for managing type 2 diabetes in primary care. Diabetes Res Clin Pract. 2017;132:169-170.

9. Shaye K, Amir T, Shlomo S, Yechezkel S. Fasting glucose levels within the high normal range predict cardiovascular outcome. Am Heart J. 2012;164(1):111-116.

10. Pyorala K, Pedersen TR, Kjekshus J, Faergeman O, Olsson AG, Thorgeirsson G. Cholesterol lowering with simvastatin improves prognosis of diabetic patients with coronary heart disease: a subgroup analysis of the Scandinavian Simvastatin Survival Study (4S). Diabetes care. 1997;20(4):614-20.

11. Briel M, Ferreira-Gonzalez I, You JJ, Karanicolas PJ, Akl EA, Wu P, et al. Association between change in high density lipoprotein cholesterol and cardiovascular disease morbidity and mortality: systematic review and metaregression analysis. Bmj. 2009;338:b92.

12. Idiopathic B, Endocrinopathies D. Report of the expert committee on the diagnosis and classification of diabetes mellitus. Diabetes care. 2003;26 suppl 1:S5-20.

13. Huang C.L, Chang H.W, Chang J.B, Chen J.H, Lin J.D, Wu C.Z, et al. Normal fasting plasma glucose predicts type 2 diabetes and cardiovascular disease in elderly population in Taiwan. QJM. 2016;109(8):515-22.

14. Bao C, Zhang D, Sun B, Lan L, Cui W, Xu G, et al. Optimal cut-off points of fasting plasma glucose for two-step strategy in estimating prevalence and screening undiagnosed diabetes and pre-diabetes in Harbin, China. PloS one. 2015;10(3):e0119510. 